\title{
Multisize and multiweight effects in materials science and engineering
}

\author{
SUN CongTing \& XUE DongFeng* \\ State Key Laboratory of Rare Earth Resource Utilization, Changchun Institute of Applied Chemistry, Chinese Academy of Sciences, Changchun \\ 130022, China
}

Received December 6, 2018; accepted December 24, 2018; published online March 15, 2019

Citation: $\quad$ Sun C T, Xue D F. Multisize and multiweight effects in materials science and engineering. Sci China Tech Sci, 2019, 62: 707-710, https://doi.org/ $10.1007 / \mathrm{s} 11431-018-9422-1$

Materials mainly refer to the matters with a certain composition, structure, and property, which can be formed by natural mineralization or artificial manufacture and are widely used in various specific fields, therefore, materials serve as the substance basis for human survival and development [1]. In the 1970s, materials combined with information and energy have been proposed as three pillars for the contemporary civilization [2]. It is obvious that materials are closely related to national economic construction, national defense construction and people's livelihood $[3,4]$. New materials, information technology and biotechnology are further recognized as the important indicators of new technological revolution in the 1980 s. Here, new materials are high knowledge intensive and high technology intensive materials with advanced physical and chemical properties [5]. In new materials industry, main challenges are the design and fabrication of the advanced materials with excellent performance, high quality as well as outstanding stability, and breaking through the limits of production scale [6].

Materials science and engineering cover the discovery, design, and fabrication of new materials [7,8]. One ultimate aim in materials research is to provide the much-needed matters to the downstream applications, such as the assembly of devices in health care, scientific study, military, communication, and so on. Design and optimization of the materials composition, structure, and properties can be individually

*Corresponding author (email: dongfeng@ciac.ac.cn) achieved in materials laboratory $[9,10]$. However, facing the downstream application industries, two aspects have to be focused on advanced materials, i.e., both multisize and multiweight effects. Materials multisize effect would satisfy different sizes requirements, and materials multiweight effect can satisfy those demands for yield in downstream applications (Figure 1). Therefore, these two key effects determine whether new materials can finally move from laboratory to industry.

Materials multisize effect refers to a variety of sizes exhibited by a certain materials ranging from micro- to macroscales $[11,12]$. Thermodynamically, materials with different sizes correspond to different energy states, which origin from the size-dependent ratio between the bulk free energy and surface free energy. That is, materials multisize effect is a single factor question. As shown in Figure 2, the system energy state decreases with the increase of material size in reactor. Our task is only to stabilize the reactor energy at a proper state. In this state, the system energy cannot provide enough driving force for the surface/interface evolution, thus materials would stop growth and retain a certain size. The energy of surface or interface at a certain state can be expressed as

$E=\left(\frac{\partial G}{\partial A}\right)_{T, P}=\frac{\sum_{i} E_{\mathrm{bond}}^{i}}{\sum_{i} A_{i}}$,

where $E$ is the energy of corresponding surface or interface, $\Delta G$ is the increased Gibbs free energy when the area of 


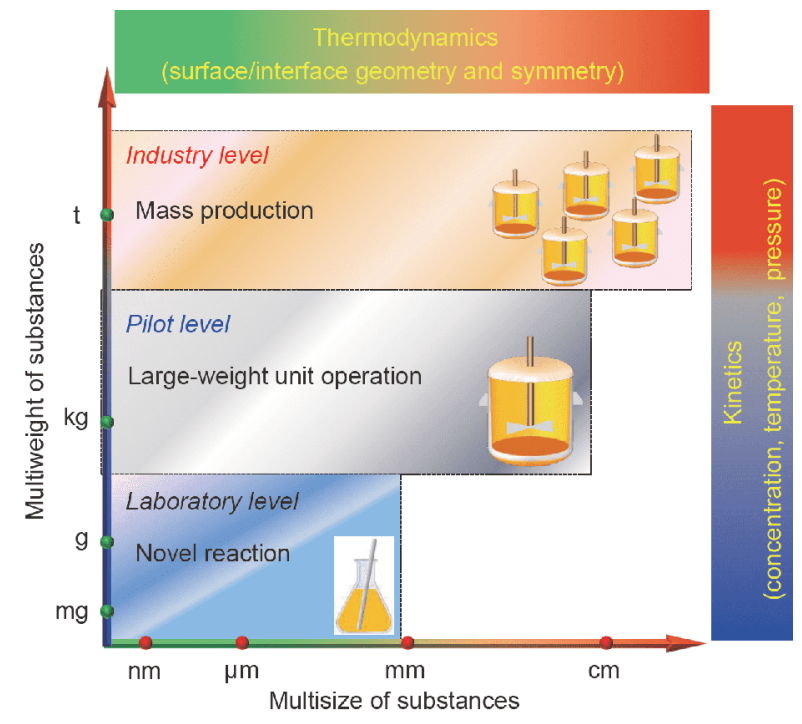

Figure 1 (Color online) Fundamentals of multisize and multiweight effects in materials science and engineering.

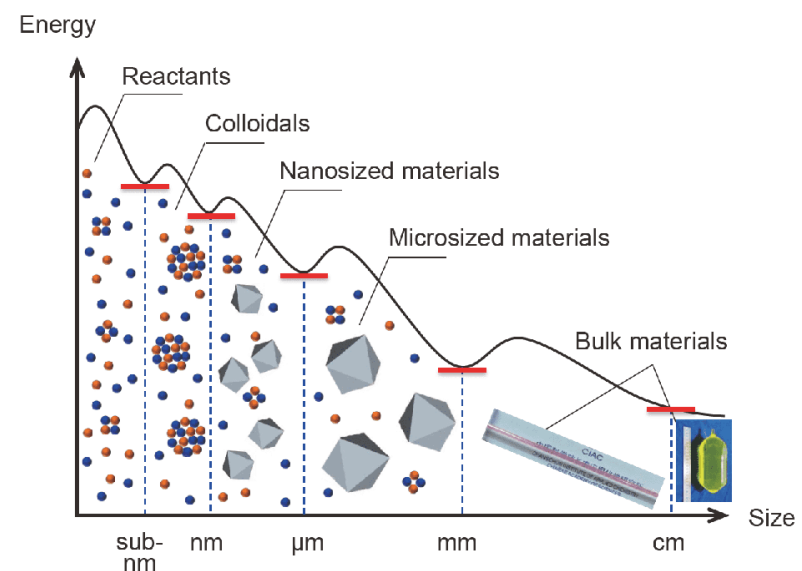

Figure 2 (Color online) Multisize effect of materials is thermodynamically determined by their monosize energy states. Individual monosize represents its surface and interface geometry, and its symmetry reflects the corresponding energy state of monosized materials.

surface or interface is increased, $A$ is the increased area of surface or interface, and $E_{\mathrm{bond}}$ is the chemical bonding energy at the surface or interface. For the monosized materials state, its Gibbs free energy can be written in terms of bulk and surface contributions, $G=G_{\text {surface }}+G_{\text {bulk }}[13]$, where $G_{\text {surface }}=$ $A \cdot \gamma$ is surface free energy, $G_{\mathrm{bulk}}=V \cdot \Delta \mu$ is bulk free energy, $\gamma$ is the unit surface free energy, and $\Delta \mu$ is chemical potential decrease when formation of unit volume substances (Figure 3). In order to enlarge the surface, the system needs to do work on the reactants, and this can move the composition to the surface/interface. Therefore, we can operate substance surface/interface to control materials multisize effect by modifying the system energy state. In material applications, the mechanical, optical, electrical and magnetic properties of advanced materials are the function of their energy states.

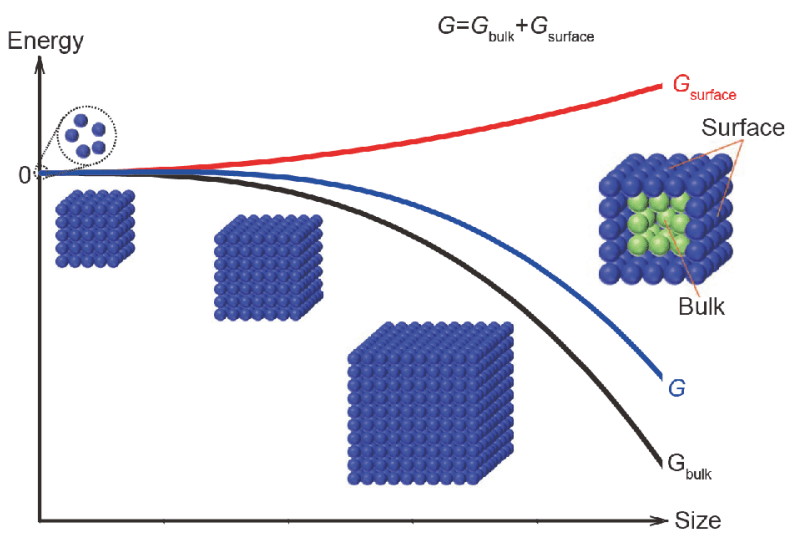

Figure 3 (Color online) Energy state of monosized materials. In order to enlarge the surface, the system needs to do work on the reactants, and this can move the composition to surface/interface.

For example, once the size of the nanomaterials is smaller than its Bohr radius, the materials will exhibit unique quantum effects, leading to materials with specific excellent properties compared to bulk materials [14]. In the reactor, surfactants with a longer carbon chain are used to hinder the system working on the surface of nanosized materials. In solid state lasers, the gain medium needs bulk optical materials such as laser single crystals, laser ceramics, and laser glasses [15]. In the fabrication of these bulk materials with the size of several millimeters, centimeters, and even decimeters, the material compositions would release amounts of heat to the environment, which promotes the system working on the surface and creates a large-size surface/interface.

Materials multiweight effect is different yields of monosized materials produced by "on pot" production, ranging from mg- to kg- and even ton-scale. Materials multiweight is a typical kinetic question, which is determined by the formation process in reactors and influenced by a series of factors, such as the reaction and transfer. In order to increase the "one pot" yield, a direct method is to scale up the reactor. The mass of reactants and corresponding reactor size are determined by thermodynamic controls. However, this will lead to multisize of the target materials due to nonuniform distribution of reactants with increasing the reactor size. The mass transfer, heat transfer, and momentum transfer need to be strengthened in a larger reactor $[16,17]$. The driving force for transfer intrinsically comes from the concentration, temperature, and velocity gradients. Because all these parameters are distance dependent, the transfer in industrial reactors is different from that in laboratory reactors. From the perspective of geometric space, the reactor cavity can be divided into a three dimensional net, which produces a large amount of nodes at the vertical and horizontal boundaries. In a certain coordinate, the vector-based representation of each node is different, resulting in distinct concentration, temperature, and velocity gradients. Inhomogeneous transfer can 
further influence the reaction in producing the materials. In order to solve these problems, dynamic controls in reactor should be clarified initially. Diffusion, reaction, and crystallization rate equations are

$J=-D \frac{\partial c}{\partial l}$

$R=k_{A} c_{A}^{\alpha} c_{B}^{\beta}$,

$v=k\left(\frac{c-c_{\mathrm{sat}}}{c_{\mathrm{sat}}}\right)^{n}$,

where $J$ is substance diffusion flux, $D$ is diffusion coefficient, $\partial c / \partial l$ is concentration gradient, $R$ is reaction rate, $k_{A}$ is reaction rate coefficient, $\alpha$ and $\beta$ are the orders of reaction, $c$ is reactant concentration, $c_{\text {sat }}$ is the saturated concentration of reactant at a certain temperature, $v$ is crystallization rate, $k$ is crystallization rate coefficient, and $n$ is the order of crystallization. According to eqs. (2)-(4), we can find that the substance reaction rate, diffusion rate, and crystallization rate equations are the functions of concentration. The reaction between reactants requires the equilibrium between diffusion rate and reaction rate. By combining eqs. (2), and (3) the diffusion-reaction equations can be established, which will give a series of numerical solutions of the concentration at a certain temperature $(T)$ since both $D$ and $k_{A}$ are $T$ dependent. Furthermore, the products will transform to crystalline state, and the concentration of products in eq. (3) determines the crystallization rate in eq. (4). The equilibrium between $R$ and $v$ can inhibit the accumulation in reactor. It is well known that $c_{\text {sat }}$ is temperature dependent. By combining eqs. (3) and (4), we will further obtain a proper temperature. Backing to the calculated results of diffusion-reaction equation, key dynamic parameters $(c, T)$ can be optimized. Such operations will produce virtual multiregime in the reactor, and each mono-regime possesses an individual uniformity (Figure 4(b)). There exist fast substance exchange and energy exchange between neighbouring mono-regimes (a)

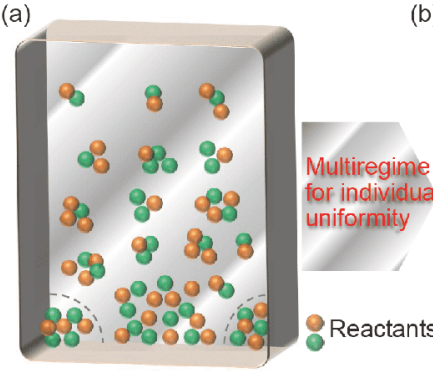

(b)

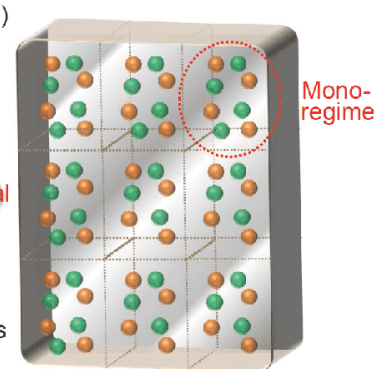

Figure 4 (Color online) Multiweight effect of monosized materials in "one pot" is dynamically determined by their kinetic controls in the reactor. (a) Substance existence state in the large-weight unit operation under thermodynamic control. The mass of reactants and corresponding reactor size are determined by thermodynamic controls. (b) Multiregime for individual uniformity under kinetic controls. By optimizing diffusion, reaction and reaction dynamics, a variety of virtual mono-regimes may be created in reactor for individual uniformity. The number and size of virtual mono-regime would be simulated by kinetic equations. to maintain the uniformity. As a consequence, materials multiweight effect can be well controlled by creating numerous microscopic reaction interfaces and designing proper parameters including concentration (pressure for gas) and temperature.

Facing the new materials industry, fundamentals in multisize and multiweight effects are inevitable key bases, which are high-knowledge intensive and high-technology intensive. In this view, multisize effect of materials is thermodynamically determined by their monosize energy states. Individual monosize represents its surface and interface geometry, and its symmetry reflects the corresponding energy state of monosized materials. Kroemer [18] proposed the concept that the interface is the device in his Nobel Lecture in 2001. In order to obtain monosized materials in "one pot" production, surface and interface fundamentals are needed to be focused on the energy state in system. On the other hand, materials multiweight effect is a kinetic question, which is closely related to substance formation process. In order to obtain the large-weight monosized materials, we need to create a variety of virtual reaction mono-regimes, in which transfer and reaction conditions are uniform. This would be established on the basis of both calculations $[19,20]$ and experiments [21,22]. In the numerical simulations, we may obtain the optimized concentration, pressure and temperature. Furthermore, step-by-step scaling up experiments would be carried out by referring those useful simulated operation parameters. Moreover, the reaction should be also optimized in size by synthetic evolution of the materials applications and economic benefits. Materials science and engineering is a multidisciplinary subject based on physics, chemistry, mechanics, and biology, which would be promoted by developing on novel concepts, theories, and technologies.

This work was supported by the National Natural Science Foundation of China (Grant Nos. 51832007, 91434118), and the National Key Research and Development Program of China (Grant No. 2016YFB0701004).

1 Bechthold M, Weaver J C. Materials science and architecture. Nat Rev Mater, 2017, 2: 17082

2 Agrawal A, Choudhary A. Perspective: Materials informatics and big data: Realization of the "fourth paradigm" of science in materials science. APL Mater, 2016, 4: 053208

3 Ali S H. The materials science imperative in meeting the sustainable development goals. Nat Mater, 2018, 17: 1052-1053

4 Foltyn S R, Civale L, Macmanus-Driscoll J L, et al. Materials science challenges for high-temperature superconducting wire. Nat Mater, 2007, 6: 631-642

5 Felser C, Fecher G H, Balke B. Spintronics: A challenge for materials science and solid-state chemistry. Angew Chem Int Ed, 2007, 46: 668699

6 Čejka J, Nachtigall P, Centi G. New catalytic materials for energy and chemistry in transition. Chem Soc Rev, 2018, 47: 8066-8071

7 Xue D F, Sun C T. 4f chemistry towards rare earth materials science and engineering. Sci China Tech Sci, 2017, 60: 1767-1768

8 Chen K, Song S, Liu F, et al. Structural design of graphene for use in 
electrochemical energy storage devices. Chem Soc Rev, 2015, 44: 6230-6257

9 Sun C T, Xue D F. Pulling growth technique towards rare earth single crystals. Sci China Tech Sci, 2018, 61: 1295-1300

10 Liang X, Chen K, Xue D. A flexible and ultrahigh energy density capacitor via enhancing surface/interface of carbon cloth supported colloids. Adv Energy Mater, 2018, 8: 1703329

11 Sun C T, Xue D F. Chemical bonding in micro-pulling down process: High throughput single crystal growth. Sci China Tech Sci, 2018, 61: 1776-1778

12 Chen K F, Xue D F. Colloidal supercapattery (in Chinese). Sci SinTech, 2019, 49: 175-181

13 Barnard A S, Curtiss L A. Prediction of $\mathrm{TiO}_{2}$ nanoparticle phase and shape transitions controlled by surface chemistry. Nano Lett, 2005, 5: 1261-1266

14 Zhang C, Li K, Song S, et al. Reversible phase transfer of luminescent $\mathrm{ZnO}$ quantum dots between polar and nonpolar media. Chem Eur J, 2013, 19: 6329-6333

15 Sun C, Xue D. Chemical bonding theory of single crystal growth and its application to $\phi 3$ " YAG bulk crystal. CrystEngComm, 2014, 16: 2129-2135

16 Huang W, Li J, Edwards P P. Mesoscience: Exploring the common principle at mesoscales. Natl Sci Rev, 2018, 5: 321-326

17 Li J, Huang W. From multiscale to mesoscience: Addressing mesoscales in mesoregimes of different levels. Annu Rev Chem Biomol Eng, 2018, 9: 41-60

18 Kroemer H. Nobel lecture: Quasielectric fields and band offsets: Teaching electrons new tricks. Rev Mod Phys, 2001, 73: 783-793

19 Ge W, Wang L, Xu J, et al. Discrete simulation of granular and particle-fluid flows: From fundamental study to engineering application. Rev Chem Eng, 2017, 33: 551-623

20 Sun C, Xue D. Crystal growth: An anisotropic mass transfer process at the interface. Phys Chem Chem Phys, 2017, 19: 12407-12413

21 Chen X, Sun C, Wu S, et al. Molecular paradigm dependent nucleation in urea aqueous solution. Cryst Growth Des, 2017, 17: 2594-2599

22 Sun C, Chen X, Xue D. Hydrogen bonding dependent mesoscale framework in crystalline $\mathrm{Ln}\left(\mathrm{H}_{2} \mathrm{O}\right)_{9}\left(\mathrm{CF}_{3} \mathrm{SO}_{3}\right)_{3}$. Cryst Growth Des, 2017, 17: 2631-2638 